\title{
Revisión
}

\section{Ingesta dietética de nitratos en bebés y niños españoles y riesgo de metahemoglobinemia}

Publicado en Internet: 14-noviembre-2013 Julio Basulto: juliobasulto@juliobasulto.com

\author{
J. Basulto ${ }^{\mathrm{a}}$, M. Manera ${ }^{\mathrm{b}}$, E. Baladia ${ }^{\mathrm{c}}$ \\ ${ }^{a}$ Nutrición Humana y Dietética. Facultad de Ciencias de la Salud y el Bienestar. \\ Universidad de Vic. Vic, Barcelona. España • bPlan integral de Promoción de la Salud mediante \\ la Actividad Física y la Alimentación Saludable (PAAS). Generalitat de Cataluña. Barcelona. \\ España • ‘Editor. Revista Española de Nutrición Humana y Dietética. España.
}

Palabras clave:

- Metahemoglobinemia

- Nitratos • Nitritos

- Verduras $\bullet$ Lactantes

- Niños
Pese a que el consumo de verduras y hortalizas es recomendable tanto en adultos como en bebés y niños pequeños, existe el riesgo de que estos últimos ingieran a través de dichos alimentos cantidades elevadas de nitratos que incrementen el riesgo de padecer metahemoglobinemia. Aunque la ingesta media de nitratos en la población infantil europea no supera los márgenes de seguridad, un análisis llevado a cabo por la Autoridad Europea de Seguridad Alimentaria (EFSA) en 2010 reveló que el consumo de espinacas puede estar implicado en ciertos casos de metahemoglobinemia infantil. La Agencia Española de Seguridad Alimentaria y Nutrición (AESAN) consideró en 2011 que las acelgas, debido a su notable consumo en España, también pueden suponer un riesgo para la población infantil si su ingesta es elevada. A las recomendaciones de la AESAN, que es conveniente que conozca cualquier profesional sanitario del ámbito de la nutrición infantil, resulta prudente añadir que un elevado consumo de borraja (Borago officinalis, una hortaliza muy consumida en Navarra, La Rioja y Aragón en la elaboración de purés hechos en casa) también puede estar implicado en la metahemoglobinemia infantil. En cualquier caso, tal y como señalan tanto la EFSA como la AESAN, cuando se comparan los riesgos/beneficios de la exposición de nitratos por el consumo de verduras y hortalizas, prevalecen los efectos beneficiosos de su consumo.

\section{Dietary intake of nitrate in Spanish infants and children and risk of methemoglobinemia}

Although the consumption of vegetables is recommended for adults, infants and young children, there is a risk that their consumption results in a high intake of nitrates that increases the risk of methemoglobinemia. Even though the average nitrate intake in children does not exceed the European safety threshold, an analysis conducted by the European Food Safety Authority (EFSA) in 2010 revealed that the consumption of spinach may be involved in certain cases of infant methemoglobinemia. The Spanish Agency for Food Safety and Nutrition (AESAN) found in 2011 that chards, because of its remarkable consumption in Spain, may also pose a risk for children. In addition to the AESAN recommendations, that any health care professional in the field of infant nutrition should know, it is prudent to add that a high consumption of borage (Borago officinalis, a vegetable widely consumed in Navarra, La Rioja and Aragón in homemade purees) may also be involved in infant methemoglobinemia. In any case, and as EFSA and AESAN point out, the benefits of eating vegetables prevail over the risks of the exposure to

- Methemoglobinemia

- Nitrates • Nitrites

- Vegetables - Infants

- Children 


\section{INTRODUCCIÓN}

El elevado contenido en nitratos de ciertas verduras muy consumidas en España puede incrementar el riesgo de que los niños menores de tres años, o aquellos con infecciones gastrointestinales, sufran metahemoglobinemia, una enfermedad potencialmente grave. Es por ello que los profesionales sanitarios implicados en la alimentación de bebés y niños deben conocer las recomendaciones actuales sobre este tema. El presente texto detalla las principales características que relacionan la metahemoglobinemia con los nitratos, incluye las actuales recomendaciones de la Agencia Española de Seguridad Alimentaria y Nutrición (AESAN) en relación con la presencia de nitratos en hortalizas y comenta un estudio llevado a cabo en España que relaciona a la borraja (Borago officinalis) con la metahemoglobinemia infantil.

\section{METAHEMOGLOBINEMIA Y NITRATOS}

En la metahemoglobinemia, la presencia de metahemoglobina en sangre está aumentada. La metahemoglobina es una forma de hemoglobina que contiene hierro férrico $\left(\mathrm{Fe}^{3+}\right)$, cuya capacidad de transportar oxígeno es menor que la de la hemoglobina ferrosa $\left(\mathrm{Fe}^{2+}\right)$, lo que se traduce en una menor llegada de oxígeno a los tejidos. Cuando la concentración de metahemoglobina en sangre alcanza cifras cercanas a un $3 \%$, se produce una cianosis, que es la principal característica de este desorden (de ahí que se conozca como "el síndrome del bebé azul").

Según el Comité de Nutrición de la Academia Americana de Pediatría, los síntomas de esta dolencia suelen ser mínimos hasta concentraciones de un 20\% de metahemoglobina. Un bebé asintomático con cianosis y una concentración de metahemoglobina inferior al $20 \%$ no suele necesitar más tratamiento que identificar y eliminar la fuente de exposición (siempre que tenga un hematocrito normal). Los niños con anemia, sin embargo, muestran toxicidad a menores concentraciones de metahemoglobina ${ }^{1}$.
Aunque existen múltiples factores implicados en esta patología ${ }^{2,3}$, uno de ellos es la ingesta de los nitratos de ciertas verduras, particularmente en bebés, como se ha apuntado en la introducción. Las bacterias de la boca y del intestino convierten los nitratos ingeridos a través de la dieta en nitritos, que reaccionan con la hemoglobina para producir metahemoglobina. Esta reacción es más brusca en menores de tres meses, debido a que una proporción de su hemoglobina está todavía en forma de hemoglobina fetal, más susceptible a la oxidación por los nitritos derivados de los nitratos. En cualquier caso, cuando la metahemoglobina ya está formada, es reconvertida en hemoglobina, tanto en adultos como en niños, mediante una enzima denominada metahemoglobina-reductasa. No obstante, esta enzima presenta una menor actividad en bebés y niños pequeños (aproximadamente la mitad que la de los adultos). Es por ello que los niños son particularmente susceptibles a la metahemoglobinemia. Esta susceptibilidad es mayor si concurre una infección bacteriana gastrointestinal, ya que se potencia la reducción de los nitratos a nitritos y la subsiguiente formación de metahemoglobina ${ }^{4}$.

\section{RECOMENDACIONES DE LA EFSA, 2010}

El Panel de Contaminantes de la Cadena Alimentaria de la Autoridad Europea de Seguridad Alimentaria (EFSA) emitió en 2008 una opinión sobre nitratos en las verduras y hortalizas, en la que determinó que la exposición a nitratos en niños y adolescentes estaba por debajo o dentro del valor de la ingesta diaria admisible (IDA). Su conclusión fue que, en general, la exposición a nitratos a partir de la ingesta de verduras y hortalizas es poco probable que resulte en un riesgo apreciable para la salud y que, por lo tanto, prevalecen los efectos beneficiosos del consumo de estos alimentos ${ }^{5}$. De hecho, existen estudios que apuntan que una dieta rica en nitratos a partir de alimentos vegetales podría ejercer beneficios sobre el sistema cardiovascular, mediante mecanismos como la disminución de la presión arterial o una protección de la disfunción endotelial, aunque faltan datos para confirmar esta hipótesis ${ }^{6-8}$. 
Dos años después del informe de la EFSA, la Comisión Europea estimó conveniente solicitar a esta entidad una evaluación de los posibles riesgos para la salud de la exposición aguda a nitratos en lactantes y niños por el consumo de dos verduras de hoja verde cuyo consumo es muy frecuente y que suelen contener elevados niveles de nitratos: la lechuga y la espinaca. En su nuevo informe, el Panel de Expertos de la EFSA tuvo en cuenta 45000 resultados analíticos de nitratos en verduras y los contrastó con el consumo real en niños de 13 países europeos. Así pues, no solo se evaluó si los niveles de nitratos en lechugas y espinacas superaban las concentraciones máximas establecidas por la legislación de la Unión Europea, sino que también se evaluó la exposición real de la población en función de su edad. Las principales conclusiones del extenso análisis de la EFSA fueron las siguientes $^{4}$ :

- La exposición crónica al nitrato de niños de 1-18 años, incluso a partir de lechuga y espinacas, no supera la IDA, lo que confirma la opinión de 2008 de este mismo organismo.

- Los bebés consumen más espinacas cocidas que lechuga. Ello, unido a que el contenido en nitratos de la lechuga es inferior al de las espinacas se traduce en que no parece existir riesgo de metahemoglobinemia a causa de la ingesta de lechuga.

- La exposición a nitratos en bebés a través de las espinacas, teniendo en cuenta la cantidad de nitratos en la media de las muestras de espinacas, no supone un riesgo. Sin embargo, sí es posible que existan bebés que tomen muchas espinacas que, a su vez, contengan más nitratos que la media. Este riesgo es alto hasta los tres años de edad del niño, pero no se puede descartar la posibilidad de cierto riesgo en algunos niños más mayores.

- Los niveles de nitratos en verduras de hoja verde son mayores cuando se cultivan en invernadero o en condiciones de iluminación reducida.

- Un almacenaje inapropiado de las verduras cocidas puede resultar en la conversión de nitra- tos a nitritos y ello supone un incremento en su potencial de causar metahemoglobinemia.

- Los bebés y niños con infecciones bacterianas del tracto gastrointestinal son más sensibles a los efectos de los nitratos, y es por ello que se desaconseja que tomen espinacas.

\section{RECOMENDACIONES DE LA AESAN, 2011}

En abril de 2011, tras tomar en consideración el citado informe de la EFSA, la AESAN emitió una serie de recomendaciones dirigidas a la población española con respecto a la presencia de nitratos en hortalizas. Pese a que ratificó las recomendaciones de la EFSA, la AESAN añadió las acelgas a la lista de hortalizas potencialmente peligrosas. Se trata de una verdura cuyo consumo en España es muy superior al observado en el resto de países europeos y cuyos niveles de nitratos pueden ser superiores a los de las espinacas. Las recomendaciones de la AESAN fueron las siguientes:

- Se recomienda, por precaución, no incluir las espinacas ni las acelgas en los purés antes del primer año de vida. En caso de incluir estas verduras antes del año, se debe procurar que el contenido de espinacas y/o acelgas no sea mayor del 20\% del contenido total del puré.

- No conviene dar más de una ración de espinacas y/o acelgas al día a niños de entre uno y tres años.

- No se debe dar espinacas y/o acelgas a niños que presenten infecciones bacterianas gastrointestinales.

- Las verduras cocinadas (enteras o en puré) no se deben mantener a temperatura ambiente. Es preciso conservarlas en el frigorífico si se van a consumir en el mismo día. Si no se van a consumir en el mismo día se deben congelar.

La AESAN insiste, en cualquier caso, en que "cuando se comparan los riesgos/beneficios de la exposición de nitratos por el consumo de hortalizas prevalecen los efectos beneficiosos reconocidos por su consumo" 9 . 


\section{ESTUDIOS PUBLICADOS DESDE LA POSTURA DE LA AESAN}

Se ha llevado a cabo una búsqueda de estudios centrados en población española o realizados en España, y publicados desde la aparición de las recomendaciones de la AESAN, en la base de datos PubMed (base de datos recomendada por el Sistema Nacional de Salud $\left.{ }^{10}\right)$. La estrategia de búsqueda ha sigo la siguiente: ("Nitrates" [Mesh] OR nitrate*) AND ("spain" [MeSH] OR "spain" [All Fields] OR "spanish" [All Fields]) AND ("2011/04/01" [PDAT]: "2013/12/31" [PDAT]).

La búsqueda desprende 255 estudios, de los cuales solo dos resultan relevantes para la presente revisión. Uno de ellos, publicado en Gaceta Sanitaria en el número de marzo-abril de 2013, evaluó los niveles de nitratos en el agua consumida en 67 municipios y 11 provincias españolas, sea del grifo o embotellada. Los niveles estuvieron, en todos los casos, dentro de los márgenes de seguridad ${ }^{11}$.

El segundo estudio aporta datos importantes para el objeto de la presente revisión. En la investigación, llevada a cabo por un equipo de científicos de Navarra y Valencia, y publicada en la revista Journal of Pediatric Gastroenterology and Nutrition, se evaluó qué hortalizas se asocian a la incidencia de metahemoglobinemia en bebés de entre 4 y 18 meses en el norte de España, mediante un estudio de casos-controles. Sus conclusiones corroboraron las recomendaciones de la AESAN, aunque añadieron que la borraja también debería considerarse dentro de las verduras cuyo consumo podría incrementar el riesgo de metahemoglobinemia. La borraja (Borago officinalis) es una hortaliza muy consumida en Navarra, La Rioja y Aragón en la elaboración de purés hechos en casa. Es posible que su acumulación de nitratos se deba, entre otros factores, al nitrógeno procedente del agua subteránea, o de diversos compuestos de nitrógeno utilizados en la agricultura.
El estudio también observó que los niños que recibían lactancia materna presentaban un riesgo de sufrir metahemoglobinemia ligeramente mayor. Ello podría explicarse por la suma de los nitratos ingeridos por la madre (que pasarían a través de la leche materna) o por la influencia de la lactancia sobre la acidez del estómago del bebé, si bien los autores consideran plausible una tercera explicación: las madres que dan el pecho son más proclives a preparar purés a sus bebés. También indican que otras investigaciones muestran el efecto contrario, es decir, que la lactancia materna protege de la metahemoglobinemia ${ }^{12}$. En el informe de la EFSA de 2008, antes citado, se señala precisamente que la lactancia materna podría proteger al bebé de la metahemoglobinemia 5 .

\section{CONCLUSIÓN}

Todo profesional sanitario implicado en la alimentación de bebés y niños debe ser conocedor de las actuales recomendaciones de la AESAN, emitidas en abril de 2011 y citadas en la presente revisión. A tales recomendaciones resulta prudente añadir la observación de un reciente estudio que ha concluido que el consumo de borraja (una hortaliza muy consumida en Navarra, La Rioja y Aragón en la elaboración de purés hechos en casa) en bebés podría incrementar su riesgo de metahemoglobinemia.

\section{CONFLICTO DE INTERESES}

Los autores declaran no presentar conflictos de intereses en relación con la preparación y publicación de este artículo.

\section{ABREVIATURAS}

AESAN: Agencia Española de Seguridad Alimentaria y Nutrición - EFSA: Autoridad Europea de Seguridad Alimentaria - IDA: ingesta diaria admisible. 


\section{BIBLIOGRAFÍA}

1. Greer FR, Shannon M; American Academy of Pediatrics Committee on Nutrition; American Academy of Pediatrics Committee on Environmental Health. Infant methemoglobinemia: the role of dietary nitrate in food and water. Pediatrics. 2005;116(3):784-6.

2. Longo D, Fauci A, Kasper D, Hauser S, Jameson J, Loscalzo J (eds.). Harrison's principles of internal medicine, 18th ed. New York: Mc Graw Hill; 2011.

3. González MP. Disminución del dolor durante la toma de muestras en niños. Rev Pediatr Aten Primaria. 2006;8:127-38.

4. European Food Safety Authority. Statement on possible public health risks for infants and young children from the presence of nitrates in leafy vegetables. EFSA J. 2010;8(12):193.

5. European Food Safety Authority. Nitrate in vegetables-Scientific Opinion of the Panel on Contaminants in the Food chain. EFSA J. 2008; 689:1-79.

6. Katan MB. Nitrate in foods: harmful or healthy? Am J Clin Nutr. 2009;90(1):11-2.

7. Machha A, Schechter AN. Inorganic nitrate: a major player in the cardiovascular health benefits of vegetables? Nutr Rev. 2012;70(6):367-72.
8. Bahra M, Kapil V, Pearl V, Ghosh S, Ahluwalia A. Inorganic nitrate ingestion improves vascular compliance but does not alter flow-mediated dilatation in healthy volunteers. Nitric Oxide. 2012;26(4):197202.

9. Agencia Española de Seguridad Alimentaria y Nutrición. Recomendaciones de consumo por la presencia de nitratos en hortalizas. 2011 [en línea] [consultado el 14/08/2013]. Disponible en www.aesan.msc.es/ AESAN/web/rincon_consumidor/subseccion/nitra tos_hortalizas.shtml

10. Grupo de trabajo sobre GPC. Elaboración de Guías de Práctica Clínica en el Sistema Nacional de Salud. Manual Metodológico. Madrid: Plan Nacional para el SNS del MSC. Instituto Aragonés de Ciencias de la Salud-I+CS; 2007. Guías de Práctica Clínica en el SNS: I+CS N.․ 2006/01.

11. Espejo-Herrera N, Kogevinas M, Castaño-Vinyals $G$, Aragonés $\mathrm{N}$, Boldo $\mathrm{E}$, Ardanaz $\mathrm{E}$, et al. Nitrate and trace elements in municipal and bottled water in Spain. Gac Sanit. 2013;27(2): 156-60.

12. Martínez A, Sánchez-Valverde F, Gil F, Clerigué N, Aznal E, Etayo V. Methemoglobinemia induced by vegetable intake in infants in northern Spain. J Pediatr Gastroenterol Nutr. 2013;56(5): 573-7. 